\title{
Validity of Parental Reporting of Recent Episodes of Acute Otitis Media: A Slone Center Office-based Research (SCOR) Network Study
}

\author{
Louis Vernacchio, MD, MSc, Richard M. Vezina, MPH, Al Ozonoff, PhD, and \\ Allen A. Mitchell, MD
}

Background: The validity of parental reporting of children's health outcomes is an important methodological issue in community-based pediatric research. We assessed the validity of parents' reports of their children's acute otitis media (AOM) history over the previous month in a pilot study of xylitol for AOM prevention.

Methods: Parents of children participating in a study conducted in the Slone Center Office-Based Research (SCOR) Network were interviewed monthly for 3 months and asked whether their child had been diagnosed with AOM in the previous month. A blinded physician reviewed medical records. Results from parental interviews and medical records were compared by correlation analysis.

Results: Medical records were obtained for 102 of 120 children (85.0\%); 272 monthly interviews were completed. $\kappa$ for the agreement between parental reports and medical records was 0.88 [95\% confidence intervals (CI): 0.76 to 0.94 ]. The positive predictive value of a parental report of an AOM episode within the previous month was $85.0 \%$, and the negative predictive value was $99.1 \%$.

Conclusions: The results of this study suggest that parental reporting of children's recent AOM history correlates well with medical records. Parental interview is a reasonable approach to collecting data on recent $\mathrm{AOM}$ outcomes, particularly in large-scale community-based studies where obtaining medical records is often impractical. (J Am Board Fam Med 2007;20:160-163.)

In community-based pediatric research, the collection of health outcomes data by medical record review can be prohibitively costly and impractical, especially for research conducted in widespread primary-care settings, where medical records are frequently hand-written and laborious to obtain. Parental reporting of children's health outcomes by interview or survey offers an alternative approach

This article was externally peer reviewed.

Submitted 27 July 2006; revised 4 October 2006; accepted 5 October 2006.

From Slone Epidemiology Center at Boston University (LV, RMV, AAM), Boston, MA; and Department of Biostatistics, Boston University School of Public Health (AO), Boston, MA.

Funding: This study was supported by the National Institute on Deafness and Other Communication Disorders, National Institutes of Health through grant R21DC058582-01.

Conflict of interest: none declared.

Previous presentation: This article is based on a presentation made at the 2006 Agency for Healthcare Research and Quality National Practice-based Research Network Conference, Bethesda, MD, May 15-17, 2006.

Corresponding author: Louis Vernacchio, MD, MSc, Slone Epidemiology Center at Boston University, 1010 Commonwealth Ave, Boston, MA 02215 (E-mail: lvernacchio@slone.bu.edu). that, if valid, would provide a cost-effective method of data collection.

To be useful, however, validity studies must be disease-specific; for example, parental reports about the occurrence of serious health conditions such as hospitalizations and cancer diagnoses are sure to have different validity than reports about mild illnesses such as routine childhood infections. ${ }^{1,2}$ Furthermore, accuracy of parental recall may vary somewhat by the child's age, birth order, and the time elapsed since the events being studied. ${ }^{1,3}$

We sought to determine the validity of parental reports of their children's acute otitis media (AOM) history over the previous month in a diverse population of subjects participating in a pilot randomized trial focused on the prevention of AOM.

\section{Methods}

In preparation for an efficacy trial of xylitol for the prevention of AOM, we conducted a pilot placebocontrolled randomized trial to asses the tolerability and acceptability of various doses of oral xylitol solution in young children. ${ }^{4}$ The study was performed in the Slone Center Office-Based Research 
(SCOR) Network, a national pediatric research network of approximately 480 pediatricians and family physicians. Physicians were recruited through a mailed invitation. In the course of their routine office practices, participating physicians in turn recruited eligible subjects to participate in the trial. Inclusion criteria were: age 6 to 36 months; history of at least 2 episodes of AOM in the previous 12 months; good general health; and Englishspeaking parent (or guardian). Exclusion criteria were: history of myringotomy tubes, intestinal malabsorption or chronic diarrhea, diabetes mellitus, any inborn errors of metabolism, and parent (or guardian) unreachable by telephone. In addition, subjects were excluded temporarily if they were currently taking any oral prescription medication or had been diagnosed with AOM in the previous 2 weeks. Informed consent was obtained by the enrolling physicians.

Subjects were randomized to receive oral xylitol or placebo solution once daily or 3 times a day for 3 months. Parents were interviewed by telephone monthly to determine tolerability of the study solution, as well as the occurrence of adverse effects, unscheduled medical visits, and hospitalizations. At least 10 attempts were made to contact parents for each interview. Parents were asked about any episodes of AOM that had been diagnosed in the previous month, who made the diagnosis, and whether antibiotic treatment was provided. The exact wording of the question was, "Since we last contacted you on [date of previous contact], has your child been seen by any doctor or nurse practitioner (for example, in an office, emergency department, health center, or clinic) for anything other than a routine checkup?" If the parent answered "Yes," then he/she was asked, "Why was your child seen?" and the following list of diagnoses was provided: "stomach pain or cramps," "excessive gas," "loose stools or diarrhea," "vomiting," "respiratory infection," "ear infection," and "other reason [specify]."

Medical records covering the time of study participation were requested from each child's primary care provider. A single blinded physician (LV) reviewed all medical records for diagnosed episodes of AOM. Episodes of AOM diagnosed outside of the children's primary care practices were disregarded, as medical records were only obtained from primary care providers. $\kappa$ statistics, adjusted for multiple interviews per parent, ${ }^{5}$ and positive and negative predictive values were calculated for the
Table 1. Characteristics of the Children Whose Medical Records Were Reviewed

\begin{tabular}{lc}
\hline Characteristic & Number $(\%)$ \\
\hline Age in months [median (range)] & $17.1(6.3-32.6)$ \\
Sex & \\
Male & $61(50.8)$ \\
Female & $59(49.2)$ \\
Ethnicity & \\
Non-Hispanic & $107(89.2)$ \\
Hispanic & $13(10.8)$ \\
Race & \\
White & $98(81.7)$ \\
Mixed Race & $7(5.8)$ \\
Black & $4(3.3)$ \\
American Indian/Alaskan Native & $2(1.7)$ \\
Unknown & $9(7.5)$ \\
State & \\
Kansas & $17(14.2)$ \\
Texas & $14(11.7)$ \\
Massachusetts & $12(10.0)$ \\
New Hampshire & $11(9.2)$ \\
Pennsylvania & $11(9.2)$ \\
Indiana & $9(7.5)$ \\
Kentucky & $9(7.5)$ \\
Oregon & $8(6.7)$ \\
South Carolina & $7(5.8)$ \\
New York & $6(5.0)$ \\
Illinois & $5(4.2)$ \\
Georgia & $3(2.5)$ \\
North Carolina & $2(1.7)$ \\
Alabama & $2(1.7)$ \\
Nebraska & $1(0.8)$ \\
West Virginia & $1(0.8)$ \\
& $1(0.8)$ \\
& $1(0.8)$ \\
\hline
\end{tabular}

agreement between parental report and the medical record. SAS version 9.1 was used for statistical analyses.

The study was approved by the Boston University School of Medicine Institutional Review Board.

\section{Results}

One hundred and twenty children from 24 primary care practices were enrolled in the study. Parentreported characteristics of the children whose medical records were reviewed are shown in Table 1. Medical records were obtained for 102 of the 120 children who participated in the xylitol pilot trial (85.0\%). All 3 monthly interviews were completed 
MEDICAL RECORD

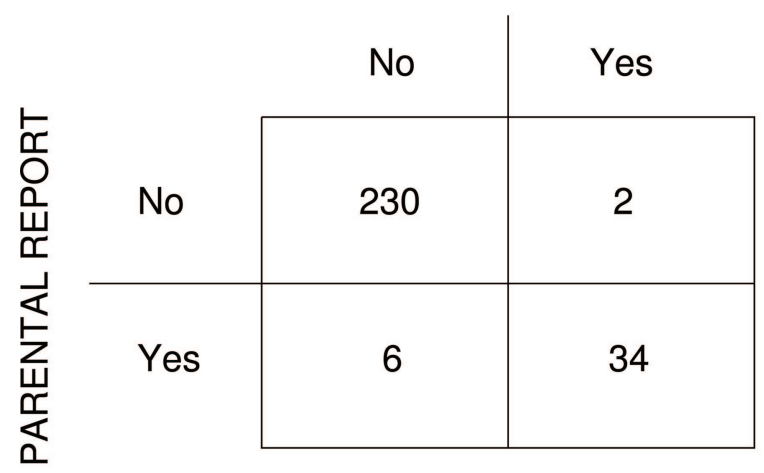

kappa $=0.88 ; 95 \% \mathrm{Cl}=0.76-0.94^{*}$

*adjusted for multiple interviews per parent

Figure 1. Agreement between parental report and medical records for young children's acute otitis media history over the previous month

by 77 parents, 2 monthly interviews were completed by 16 parents, and 1 monthly interview was completed by 9 parents, for a total of 272 interviews.

Parents reported that an AOM episode was diagnosed at the primary care provider's office in the previous month during 40 of the 272 interviews (14.7\%), and an antibiotic was prescribed for 39 of the 40 episodes $(97.5 \%)$. Parents reported an additional 4 AOM episodes diagnosed outside the primary care provider's office, which were disregarded for the current analysis. The parental report was in complete agreement with the medical record for 264 of the interviews (97.1\%). During 6 interviews, parents reported an AOM episode diagnosed at the primary care provider's office that was not found in the medical record (2.2\%). In 2 interviews, a parent reported that their child had not had an AOM episode although one was found in the medical record $(0.7 \%)$. The $\kappa$ statistic for agreement between the parent and the medical record, adjusted for the effect of multiple interviews per parent, ${ }^{5}$ was 0.88 with a $95 \%$ confidence interval of 0.76 to 0.94 (Figure 1). The positive predictive value of a parental report of an AOM episode within the previous month was $85.0 \%$, and the negative predictive value was $99.1 \%$.

\section{Discussion}

In the context of a pilot study about AOM prevention, we found parents' reports of their children's recent AOM history over the previous month to correlate extremely well with the medical record, with a $\kappa$ value of 0.88 . Of the few discrepancies found in our study, most ( 6 of the 8 errors) were the result of parental over-reporting of AOM episodes. Over-reporting may occur when a parent misunderstands a physician's diagnosis to be AOM when it is in fact something else (for example, otitis media with effusion or simple upper respiratory tract infection). We found evidence of this phenomenon in 2 of the 6 false positive parental reports in our study. It is also possible that, in cases of overreporting, the parental report is actually correct, and the medical record is incomplete or inaccurate, although we were not able to evaluate this possibility. Although the medical record has traditionally been seen as the "gold standard" for data collection in clinical research, in some cases, parental report may be indeed more accurate.

Our findings are similar to those from a study conducted among a more geographically and socioeconomically homogenous population of Minnesota parents. Among those parents, a $\kappa$ value of 0.94 was found for parental recall of children's AOM history over the previous 3 months. ${ }^{6}$ However, the accuracy of parental reporting beyond 3 months' time has been shown to be lower; recall over 1 to 2 years is only fair, with increasing instances of both parental over-reporting and under-reporting given such time frames. 3

The results of this study suggest that parental reporting of children's recent AOM history correlates well with medical records. Parental interview is therefore a reasonable approach to collecting data on recent AOM outcomes, particularly in large-scale or multisite community-based studies in which obtaining medical records is costly and impractical.

We thank the following members of the SCOR Network who participated in the study: Charles Anderson, MD (San Marcos, TX), Barbara P. Belcher, MD (San Antonio, TX), Michael A. Blum, DO (Overland Park, KS), Paul M. Douthitt, MD (Springfield, TN), Patricia Edwards, MD (Concord, NH), Michael F. Grossberg, MD (Chambersburg, PA), Daniel E. Halm, MD (Bellevue, NE), James Horwitz, MD (Hendersonville, NC), Shahina R. Khan, MD (Gaffney, SC), George M. McCormick, MD (State College, PA), John Mulawka, D.O. (Silver Creek, NY), Sree K. Mulpuru, MD (Fairmont, WV), Beth Nauert, MD (Austin, TX), Jo Anne Nielsen, MD (Oregon City, OR), Jagdish Patel, MD (Bronx, NY), Suzanne W. Schuessler, MD (Lagrange, GA), Onkar Sharma, MD (Mattoon, IL), Merl W. Simmons, MD (Oklahoma City, OK), Michael W. Simon, MD 
(Lexington, KY), Lyle D. Smith, MD (Dodge City KS), Neil J. Stalker, MD (Peru, IN), Jeb S. Teichman, MD (Jeffersonville, IN), Louis Vernacchio, MD (Boston, MA), Shaila N. Williston, MD (Orange Park, FL), and Michael F. Yeiser, MD (Owensboro, $\mathrm{KY})$.

\section{References}

1. D'Souza-Vazirani D, Minkovitz CS, Strobino DM. Validity of maternal report of acute health care use for children younger than 3 years. Arch Pediatr Adolesc Med 2005;159:167-72.

2. Cooper WO. Validity of maternal report of acute health care for children: evaluating proxy interview responses. Arch Pediatr Adolesc Med 2005;159: 193-4.

3. Pless CE, Pless IB. How well they remember. The accuracy of parent reports. Arch Pediatr Adolesc Med 1995;149:553-8.

4. Vernacchio L, Vezina RM, Mitchell AA. Tolerability of oral xylitol solution in young children: implications for otitis media prophylaxis. Int J Pediatr Otorhinolaryngol 2007;71(1):89-94.

5. Donner A, Klar N. The statistical analysis of kappa statistics in multiple samples. J Clin Epidemiol 1996; 49:1053-8.

6. Daly KA, Lindgren B, Giebink GS. Validity of parental report of a child's medical history in otitis media research. Am J Epidemiol 1994;139:1116-21.

7. Alho OP. The validity of questionnaire reports of a history of acute otitis media. Am J Epidemiol 1990; 132:1164-70. 